\title{
Repair bond strength of microhybrid, nanohybrid and nanofilled resin composites: effect of substrate resin type, surface conditioning and ageing
}

\author{
Mutlu Özcan • Pedro Henrique Corazza • \\ Susana Maria Salazar Marocho • \\ Silvia Helena Barbosa • Marco Antonio Bottino
}

Received: 19 September 2011 / Accepted: 10 October 2012 /Published online: 19 October 2012

(C) Springer-Verlag Berlin Heidelberg 2012

\begin{abstract}
Objectives This study evaluated the microtensile bond strength (MTBS) of non-aged and aged resin-based composites (RBC) (nanohybrid and nanofilled) after two surface conditioning methods, repaired using the composite of the same kind or a microhybrid composite.

Materials and methods Nanohybrid (Tetric EvoCeramTE) and nanofilled (Filtek Supreme-FS) RBC blocks (5× $5 \times 6 \mathrm{~mm})(N=128)$ were fabricated and randomly divided into two groups: (a) no ageing (control group) and (b) ageing $\left(5.000\right.$ thermocycling, $\left.5-55^{\circ} \mathrm{C}\right)$. RBC surfaces were polished by up to 1,200-grit silicone carbide papers and conditioned with either (a) air abrasion with $30-\mu \mathrm{m} \mathrm{SiO}_{2}$ particles (CoJet Sand) for $4 \mathrm{~s}+$ silane coupling agent (ESPE-Sil) + adhesive resin (VisioBond) $(n=16)$ or (b) adhesive application only (Multilink A+B for TE; Adper ScotchBond 1XT for FS) $(n=16)$. In half of the groups, repair resin of the same kind with the RBC and, in the other half, a different kind of composite (microhybrid, Quadrant Anterior Shine-AS) with its corresponding adhesive (Quadrant UniBond) was used. The specimens were submitted to MTBS test $(0.5 \mathrm{~mm} / \mathrm{min})$. Data were analysed
\end{abstract}

M. Özcan ( $\square)$

Dental Materials Unit, Center for Dental and Oral Medicine,

Clinic for Fixed and Removable Prosthodontics and Dental

Materials Science, University of Zürich,

Plattenstrasse 11,

8032 Zürich, Switzerland

e-mail: mutluozcan@hotmail.com

P. H. Corazza - S. M. S. Marocho - S. H. Barbosa • M. A. Bottino

Department of Dental Materials and Prosthodontics,

Sao Paulo State University,

Av. Francisco José Longo, 777,

12245-000 Sao Jose dos Campos, Brazil using three-way ANOVA and Tukey's tests. Degree of conversion (DC) of non-aged and aged resin composites (TE, FS) ( $n=3$ per group) was measured by micro-Raman analyses.

Results RBC type $(p=0.001)$ and ageing affected the MTBS results significantly $(p=0.001)$. Surface conditioning type did not show significant difference $(p=0.726)$, but less number of pre-test failures was experienced with the CoJet system compared to adhesive resin application only. Repair strength on aged TE showed significantly less $(p<0.05)$ MTBS than for FS. FS repaired with the same kind of $\mathrm{RBC}$ and adhesive resin presented the highest cohesive failures $(43 \%)$. DC was higher for TE (71\%) than for FS (58\%) before ageing.

Conclusion On the aged RBCs, less favourable repair strength could be expected especially for nanohybrid composite. For repair actions, RBC surface conditioning could be accomplished with either adhesive resin application only or with CoJet system, providing that the latter resulted in less pre-test failures.

Clinical relevance Clinicians could condition the resin surface prior to repair or relayering with either CoJet system or adhesive resin application only, depending on the availability of the system.

Keywords Ageing $\cdot$ Microtensile bond strength $\cdot$ Minimal invasive dentistry $\cdot$ Repair $\cdot$ Resin composite $\cdot$ Surface treatment

\section{Background}

Advances in filler technology improved the properties of resin-based composites (RBC) used in dentistry. These 
developments yielded to the introduction of nanohybrid and nanofilled RBCs $[1,2]$. While the size of the filler particles lie around $8-30 \mu \mathrm{m}$ in hybrid composites and $0.7-3.6 \mu \mathrm{m}$ in microhybrid composites, RBCs with nanofillers exhibit filler sizes ranging from 5 to $100 \mathrm{~nm}[1,2]$. Yet, nanofilled RBCs still exhibit clinical problems such as secondary caries and fractures in the long term [3-5]. A great conservative alternative to manage deficient $\mathrm{RBC}$ restorations is the repair. Repair procedures are mainly justified in terms of entailing removal of enamel and/or dentin leading to more loss of sound dental tissues [6,7], reduction of the potential harmful effects on the pulp, reducing treatment expenses and treatment time, and increasing longevity of restorations $[6,7]$.

Repair protocols demonstrated substantial difficulty in establishing a reliable bond to the existing "aged" composite for RBC $[8,9]$. Despite the presence of unreacted methacrylate groups after the polymerization, the amount of unsaturated double bonds diminishes with ageing and reduces the resin adhesion potential [10-13]. Different periods of water storage and thermocycling are some of the methods used to simulate the ageing process of dental materials in in vitro studies $[8,9,13]$. These methods attempt to simulate the hydrolytic degradation occurring in $\mathrm{RBC}$ restorations in service. On the other hand, it is difficult for the clinician to know the nature of the RBC especially when the patient is referred from other practices. This factor coupled with the aged surface properties requires conditioning RBC surfaces prior to repair [14].

Several surface conditioning methods have been recommended to improve the repair bond strength, such as surface roughening with diamond burs, etching with hydrofluoric or phosphoric acid etching, or airborne abrasion with aluminium oxide particles or aluminium oxide particles coated with silica [11, 13-21]. Roughening methods provide surface irregularities, promoting micro-mechanical interlocking between the substrate surface and the repair resin, but these procedures are further associated with the application of wetting agents such as silane coupling agents and adhesive resins $[17,22]$. The use of adhesive wetting agents promotes chemical adhesion between the repair resin and the substrate $[21,22]$.

The surface conditioning based on silica coating followed by silanization and adhesive resin application, called tribomechanical silica coating, was initially used for conditioning metal alloys for ceramic repairs and for conditioning cementation surfaces of metal reconstructions [23, 24]. The technique requires additional armamentarium in the clinical settings that adds to the cost of the treatment compared to application of an adhesive resin only. Tribomechanical silica coating has been tried for repairs of conventional RBC materials $[9,14,25]$ but rarely studied for nanohybrid or nanofilled RBCs [26, 27]. Moreover, resin repair protocols have not been well established for situations where the substrate and the adherend were not of the same kind $[28,29]$.

The objectives of this study, therefore, were to (a) compare the repair bond strength of non-aged and aged RBCs after two surface conditioning methods repaired using either the composite of the same kind with the substrate or a different one and (b) measure the degree of conversion of the used substrate RBCs before and after ageing.

\section{Materials and methods}

The brand, type, chemical compositions and manufacturers of the materials used in this study are listed in Table 1.

\section{Specimen preparation}

Nanohybrid (Tetric EvoCeram-TE, shade A2) and nanofilled (Filtek Supreme-FS, shade A2) RBC blocks $(5 \times 5 \times$ $6 \mathrm{~mm})(N=128)$ were built up incrementally using a silicone mould. Each layer was photo-polymerized (XL3000, 3M ESPE, St. Paul, MN, USA) following each manufacturer's recommended time. Light intensity was assured to be higher than $400 \mathrm{~mW} / \mathrm{cm}^{2}$, verified by a radiometer after every five specimens (Demetron LC, Kerr, Orange, CA, USA). Each specimen was removed from the mould and ground finished with silicone carbide papers of 120, 400, 600, 800 and 1,200 grit in sequence at $300 \mathrm{rpm}$ under water irrigation. They were then randomly divided into two groups: (a) no ageing (control group) $(n=64)$ and (b) ageing (5.000 thermocycling, $5-55^{\circ} \mathrm{C}$ ) $[14,27,29]$ (Nova Etica, São Paulo, Brazil) $(n=64)$. Control group specimens were kept in dry conditions in the dark until the experiments.

Half of the non-aged and aged specimens were air abraded with $30-\mu \mathrm{m} \mathrm{SiO}{ }_{2}$ particles (CoJet Sand, 3M ESPE, Seefeld, Germany) under 2.8 bar pressure from a distance of approximately $10 \mathrm{~mm}$ for $4 \mathrm{~s}$. Silane coupling agent (ESPE-Sil, 3M ESPE) was applied, and the reaction was awaited for $5 \mathrm{~min}$. Adhesive resin (VisioBond, 3M ESPE) was applied with a microbrush, air thinned and photo-polymerized for $20 \mathrm{~s}$. In the other half, only the corresponding adhesive resins of the RBCs were applied (Multilink A+B for TE; Adper ScotchBond 1XT for FS) applied with a microbrush, air thinned and photopolymerized for $20 \mathrm{~s}$. In these groups, the repair composite used was of the same kind as the substrate (TE-TE, FS-FS).

In order to simulate the situation where the substrate material cannot be clinically identified and a dissimilar material has to be used, in half of the groups, a different kind of microhybrid RBC (Quadrant Anterior Shine-AS, Cavex, Haarlem, the Netherlands) (TE-AS, FS-AS) with its corresponding adhesive resin (Quadrant UniBond, Cavex) was used. The adhesive was applied with a microbrush, air 
Table 1 The brand, type, chemical compositions and manufacturers of the materials used in this study

\begin{tabular}{|c|c|c|}
\hline Brand, type & Chemical composition & Manufacturer \\
\hline $\begin{array}{l}\text { Tetric EvoCeram (TE) } \\
\text { (nanohybrid) }\end{array}$ & $\begin{array}{l}\text { bis-GMA and TEGDMA, dimethacrylate-based monomers }(17-18 \% \\
\text { weight), barium glass, ytterbium trifluoride, mixed oxide and prepoly- } \\
\text { mer containing fillers }(82-83 \% \text { weight), additives, catalysts, stabilizers } \\
\text { and pigments }(<1.0 \% \text { weight). The particle size of the inorganic fillers } \\
\text { is between } 40 \text { and } 3,000 \mathrm{~nm} \text { with a mean particle size of } 550 \mathrm{~nm} \text {. }\end{array}$ & $\begin{array}{l}\text { Ivoclar Vivadent, Schaan, } \\
\text { Liechtenstein }\end{array}$ \\
\hline $\begin{array}{l}\text { Filtek Supreme XT (FS) } \\
\text { (nanofilled) }\end{array}$ & $\begin{array}{l}\text { bis-GMA, TEGDMA, UDMA, ethoxylated bisphenolglycidyl } \\
\text { methacrylate (bis-EMA), zirconia-silica, silica-containing fillers } \\
(57.7 \mathrm{vol} \%)\end{array}$ & $\begin{array}{l}\text { 3M ESPE, Seefeld, } \\
\text { Germany }\end{array}$ \\
\hline $\begin{array}{l}\text { Quadrant anterior shine (AS) } \\
\text { (microhybrid) }\end{array}$ & $\begin{array}{l}\text { Methacrylate-based monomers, } 23.7 \mathrm{wt} \% \text {; silica-, silicate glass- and } \\
\text { fluoride-containing fillers, } 75.6 \mathrm{wt} \% \text {; polymerization catalysts, } \\
0.6 \mathrm{wt} \% \text {; inorganic pigments, } 0.1 \mathrm{wt} \%\end{array}$ & $\begin{array}{l}\text { Cavex, Haarlem, the } \\
\text { Netherlands }\end{array}$ \\
\hline CoJet Sand & Aluminium trioxide particles coated with silica; particles size, $30 \mu \mathrm{m}$ & 3M ESPE \\
\hline ESPE-Sil & 3-Methacryloxypropyltrimethoxysilane, ethanol & 3M ESPE \\
\hline VisioBond & $\begin{array}{l}\text { Bisacrylate, aminodiol methacrylate, camphor quinone, benzyl dimethyl } \\
\text { ketale, stabilizers }\end{array}$ & 3M ESPE \\
\hline Multilink A+B & $\begin{array}{l}\text { Primer A: water, initiators (sulfonate, amines); primer B: phosphonic acid } \\
\text { acrylate, HEMA, TEGDMA, methacrylate modified polyacrylic acid }\end{array}$ & Ivoclar Vivadent \\
\hline Adper Scotchbond 1XT & $\begin{array}{l}\text { Dimethacrylate, HEMA, polyalcenoic acid copolymer, silane-treated col- } \\
\text { loidal silica, ethanol, water, photoinitiator }\end{array}$ & 3M ESPE \\
\hline Quadrant Unibond & $\begin{array}{l}\text { bis-GMA, TEGDMA, silicate glass fillers, silica, polycarboxylic acid, } \\
\text { champorquinone }\end{array}$ & Cavex \\
\hline
\end{tabular}

thinned and photo-polymerized for $20 \mathrm{~s}$. Thus, eight blocks $(n=8)$ were obtained per group and tested.

Experimental groups are schematically presented depending on the composite-composite combinations, ageing and surface conditioning methods in Fig. 1.

\section{Microtensile bond test}

Bonded RBC blocks were sectioned with a diamond saw in a precision cutting machine at low speed, under water cooling (Labcut 1010; Extec Corp., Enfield, CT, USA). Initially, the blocks were glued with cyanoacrylate adhesive (Super Bonder Gel, Loctite Ltd., São Paulo, Brazil) on a metallic base that was attached to the sectioning machine. The blocks were positioned as perpendicular as possible in relation to the diamond disc of the machine. The peripheral slices, measuring approximately $0.5 \mathrm{~mm}$, were discarded in case the results could be influenced by either the excess or insufficient amount of resin at the margins. Thus, only the central specimens were used for the experiments. Nontrimmed rectangular beams with an adhesive area of $1 \pm$ $0.1 \mathrm{~mm}^{2}$ and a length of about $10 \mathrm{~mm}$ were achieved from each block. From each block, 9 and, for each group, 72, beams were planned to be obtained. The debonded beams during cutting procedures were considered as pre-test failures. Pre-test failures were not involved in the microtensile bond test (MTBS) calculations.

Before testing, the adhesive area of each beam was measured using a digital caliper (Mitutoyo, Tokyo, Japan). Each specimen was bonded with cyanoacrylate adhesive to a custom-made adapted caliper perpendicular to the force applied in order to avoid shear forces at the interface. Only the ends of the specimens were bonded. The device/specimen assembly was adapted to the Universal Testing Machine (EMIC DL 1000; EMIC, São José dos Pinhais, PR, Brazil), and beam specimens were tested in microtensile strength at a crosshead speed of $0.5 \mathrm{~mm} / \mathrm{min}$ until fracture. The bond strength $\sigma$ (in megapascals) was calculated
Fig. 1 Schematic presentation of the experimental groups depending on the substraterepair resin type, ageing conditions and surface conditioning methods used

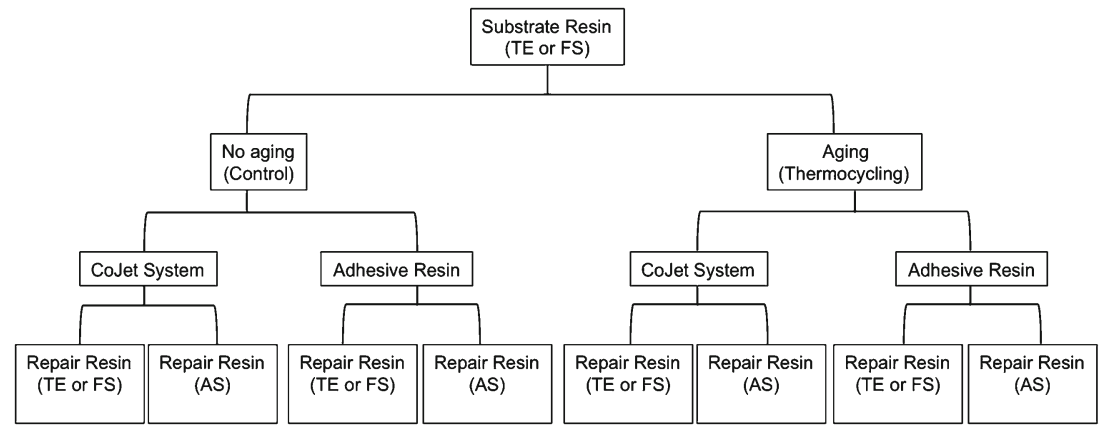


according to the formula $\sigma=L / A$, where $L$ is the load for rupture of the beam specimen and $A$ is the interfacial area (in square millimetres).

Failure type analysis

After MTBS, all specimens were analysed using an optical microscope (Wild M7, Heerbrugg AG, Heerbrugg, Switzerland) at $\times 30$ magnification for failure analysis. Failure types were categorized as adhesive between the substrate-adherend interface and cohesive failure of the RBC.

A cold field emission scanning electron microscope (SEM) (JSM 6301F, Jeol Instruments, Tokyo, Japan) was used to observe the aged and non-aged specimen surfaces. Images were made at $25 \mathrm{kV}$ at a magnification of $\times 5,000$ and $\times 15,000$. Surfaces were first sputter-coated with gold/ palladium $(80 / 20)$ prior to examination.

\section{Degree of conversion}

Additional specimens from non-aged and aged TE and FS blocks ( $N=9, n=3$ per group) were built up as described above. Degree of conversion (DC) was measured at room temperature using a Raman microscope (System 2000, Renishaw, IL, USA). Each spectrum of unpolymerized RBC was obtained between 1,400 and $-1,800 \mathrm{~cm}^{-1}$.

The DC was calculated according to the following equation:

$\mathrm{DC}(\%)=100 \times\left[1-\left(\mathrm{R}_{\text {polymerized }} / \mathrm{R}_{\text {unpolymerized }}\right)\right]$

where $R=$ band height at $1,650 \mathrm{~cm}^{-1} / \mathrm{band}$ height at $1,616 \mathrm{~cm}^{-1}$.

\section{Statistical analysis}

Statistical analysis was performed using the software Statistix 8.0 for Windows (Analytical Software Inc, Tallahassee, FL, USA). The data were submitted to three-way analysis of variance (ANOVA) with MTBS data (in megapascals) as the dependent variable and RBC type (nanohybrid versus nanofilled), ageing conditions (two levels; non-aged versus aged) and conditioning methods (two levels; silica coating versus adhesive resin) as independent variables. Multiple comparisons were made using Tukey's test. The $p$ values less than 0.05 were considered to be statistically significant in all tests.

\section{Results}

RBC type ( $p=0.001)$ and ageing affected the MTBS results significantly $(p=0.001)$. Surface conditioning type did not show significant difference $(p=0.726)$ (Table 2).
Repair strength for aged TE $(33 \pm 8.7-40.5 \pm 13.3)$ showed significantly less $(p<0.05)$ MTBS than for FS $(44 \pm 15.8-48.1 \pm$ 12.3 ) with both conditioning methods (Table $3 a$ and $b$ ). The use of a dissimilar repair resin (TE-AS, FS-AS) did not significantly affect the results in both non-aged and aged conditions $(p=0.922)$.

Less number of pre-test failures was experienced with the CoJet system $\left(n_{\text {beam }}=162\right)$ compared to adhesive resin $\left(n_{\text {beam }}=142\right)$ application only.

FS repaired with the same kind of RBC (FS-FS) and conditioned with its corresponding adhesive resin presented the higher incidence of cohesive failures (43\%) than TE under the same conditions (TE-TE) (10\%) (Fig. 2). DC was higher for TE (71 \%) than for FS (58 \%) but did not change significantly after ageing (68 and $61 \%$ for TE and FS, respectively). SEM images of aged specimens showed surface degradation of both TE and FS compared to the nonaged baseline situation (Fig. 3a-d).

\section{Discussion}

RBCs are routinely used in dentistry to restore damaged teeth due to caries or trauma and to repair damaged restorations in a minimally invasive fashion. Indications for repair include the need to correct marginal defects, surface discoloration, partial loss, abrasion or attrition, and bulk fracture of anterior and posterior restorations [7]. In this study, two clinical situations were simulated, one being the situation where the clinician repairs the substrate RBC with that of the same material and the second where the nature of the substrate RBC is not known and therefore a dissimilar material was selected.

During incremental layering, an oxygen-inhibited layer makes reliable adhesion of subsequent increments possible $[10,30]$. Adhering a new RBC to an existing one presents a different challenge because of the absence of an oxygeninhibited layer. Hence, in order to ensure adhesion of a new $\mathrm{RBC}$ to an existing one, the surface of the substrate material needs to be activated either by adhesive resins in a chemical way or using other methods that are based on physicochemical conditioning methods. Previous studies reported that due to the reduction of unreacted methacrylate monomers over time in clinical function and the intervention with polishing instruments, repair bond may be impaired [13, $14,20,21]$. According to the results of this study, in nonaged conditions, the two RBCs used in this study behaved similarly. However, repair bond strength of nanofilled composite FS showed decreased repair strength after thermocycling, yet not significant. On the other hand, nanohybrid TE showed decreased bond strength after thermocycling with some repair protocols. This could be justified by the fact that nanohybrid TE contains a resin matrix composed of bis- 
Table 2 Results of three-way ANOVA and Tukey's test and interactions considering the substrate composite type, ageing and surface conditioning, using different adherends

$* p<0.05$

\begin{tabular}{llllll}
\hline Source & DF & SS & MS & F & P \\
\hline Resin composite type & 1 & 0.37279 & 0.36005 & 17.03 & $0.001^{*}$ \\
Ageing & 1 & 1.11313 & 0.95827 & 45.33 & $0.001^{*}$ \\
Surface conditioning & 1 & 0.00128 & 0.00260 & 0.12 & 0.726 \\
Resin composite $\times$ ageing & 1 & 0.16641 & 0.14048 & 6.65 & $0.010^{*}$ \\
Resin composite $\times$ surface conditioning & 1 & 0.01227 & 0.00177 & 0.08 & 0.773 \\
Ageing $\times$ surface conditioning & 1 & 0.040066 & 0.02519 & 1.19 & 0.275 \\
Resin composite $\times$ ageing $\times$ surface conditioning & 1 & 0.00802 & 0.00557 & 0.26 & 0.608 \\
Substrate-adherend difference & 64 & 1.01759 & 0.01590 & 0.75 & 0.922 \\
Error & 548 & 11.58493 & 0.02114 & & \\
Total & 626 & 14.44474 & & & \\
\hline
\end{tabular}

GMA and TEGDMA, whereas nanofilled FS contains bisGMA, bis-EMA, UDMA and TEGDMA. bis-GMA leads to the formation of a rigid cross-linked network (1.43 GPa) that absorbs less water than TEGDMA but higher than those of UDMA and bis-EMA [31]. TEGDMA is a hydrophilic monomer, meaning that it absorbs greater amounts of water $[32,33]$.

Ageing of RBCs is often as a consequence of mechanical/physical degradation mechanisms such as wear, abrasion and fatigue or due to chemical degradation mechanisms such as enzymatic, hydrolytic, acidic or temperaturerelated breakdown [32]. One common route of simulating the hydrothermal ageing is exposing the specimens to thermocycling. The alternating temperatures between 5 and $55^{\circ}$
$\mathrm{C}$ coupled with the presence of water contribute to ageing of RBC. Previous studies showed the ageing effect of thermocycling and long-term water storage on the substrate compared to other ageing methods such as storage in citric acid [14, 27, 29]. The water absorption has a detrimental effect on the physical and structural integrity of some RBCs, including flexural and bond strength. Decreased cohesive strength of the material due to the hydrolytic degradation through the oxidation process and/or hydrolysis was reported [32]. One recent study showed even more ageing effect of the presence of biofilm [34]. However, previous studies only used shear test while making this judgement [27-30, 34]. Shear tests have been criticized for the development of non-homogeneous stress distributions in the
Table 3 Mean microtensile bond strength results for TE (a) and FS (b) repaired with the same and dissimilar composites

The same letters in one column indicate no significant differences (Tukey's test, $p<0.05$ ). See Table 1 for material abbreviations

\begin{tabular}{|c|c|c|c|c|}
\hline Ageing & $\begin{array}{l}\text { Substrate-adherend } \\
\text { combinations }\end{array}$ & $\begin{array}{l}\text { Surface } \\
\text { conditioning }\end{array}$ & $n_{\text {beams }}$ & Mean (SD) \\
\hline \multicolumn{5}{|l|}{$\mathrm{a}$} \\
\hline \multirow[t]{4}{*}{ No ageing (control) } & \multirow[t]{2}{*}{ TE-AS } & CoJet & 44 & 48.1 (14.1) A, B \\
\hline & & Adhesive resin & 40 & 48.7 (15.8) A, B \\
\hline & \multirow[t]{2}{*}{ TE-TE } & CoJet & 52 & $47.6(13.2) \mathrm{A}, \mathrm{B}$ \\
\hline & & Adhesive resin & 32 & $54.1(17.2) \mathrm{A}$ \\
\hline \multirow[t]{4}{*}{ Ageing (thermocycling) } & \multirow[t]{2}{*}{ TE-AS } & CoJet & 31 & $37.9(8.4) \mathrm{C}$ \\
\hline & & Adhesive resin & 39 & 40.5 (13.3) B, C \\
\hline & \multirow[t]{2}{*}{ TE-TE } & CoJet & 38 & $39.6(9.6) \mathrm{B}, \mathrm{C}$ \\
\hline & & Adhesive resin & 40 & $33(8.7) \mathrm{C}$ \\
\hline \multicolumn{5}{|l|}{$\mathrm{b}$} \\
\hline \multirow[t]{4}{*}{ No ageing (control) } & \multirow[t]{2}{*}{ FS-AS } & CoJet & 42 & $52.5(15.6) \mathrm{A}$ \\
\hline & & Adhesive resin & 38 & $51(16) \mathrm{A}$ \\
\hline & \multirow[t]{2}{*}{ FS-FS } & CoJet & 39 & $51.5(13.5) \mathrm{A}$ \\
\hline & & Adhesive resin & 23 & $54(21.1) \mathrm{A}$ \\
\hline \multirow[t]{4}{*}{ Ageing (thermocycling) } & \multirow[t]{2}{*}{ FS-AS } & CoJet & 31 & $48.1(12.3) \mathrm{A}$ \\
\hline & & Adhesive resin & 44 & 47.3 (12.7) A \\
\hline & \multirow[t]{2}{*}{ FS-FS } & CoJet & 50 & $45.1(10.5) \mathrm{A}$ \\
\hline & & Adhesive resin & 37 & $44(15.8) \mathrm{A}$ \\
\hline
\end{tabular}


Fig. 2 Percentage of cohesive failures experienced in substrate-repair resin combinations after microtensile bond test. Cont control, TC thermocycling, $C J$ CoJet, $A D$ adhesive

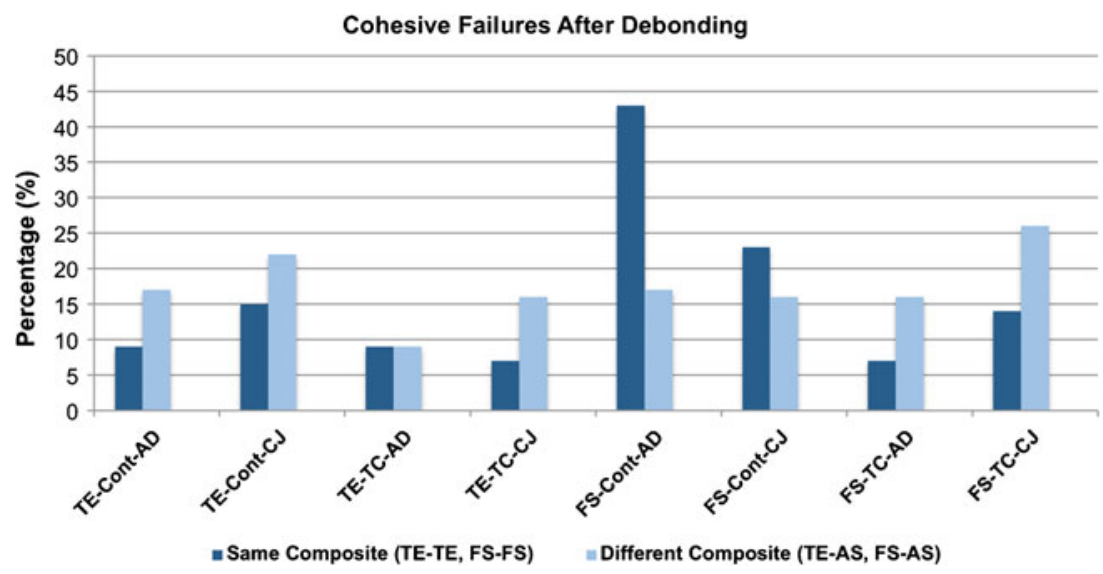

bonding interface, inducing either an underestimation or a misinterpretation of the results, since failure often starts in one of the substrates and not at the adhesive zone [35]. For this reason, in this study, MTBS test was used. Analysis of SEM images after ageing has shown deterioration in the RBC surfaces being more significant for TE. Thus, one may suspect that this structural damage may affect the bonding ability of the RBC to be repaired. Complimentary DC tests aimed to compare the results with previous studies $[27,36,37]$, and the values were not significantly less after thermocycling for both materials tested. This puts the real ageing effect of thermocycling in question. DC expresses the amount of remaining carbon double bonds $(\mathrm{C}=\mathrm{C})$ after polymerization that are located in the end of the polymer chains or lost into the RBC volume [37]. The ageing process might be able to leach the $\mathrm{C}=\mathrm{C}$ resulting from loose monomers or oligomers, but the $\mathrm{DC}$ based on the $\mathrm{C}=\mathrm{C}$ at the end of the chains will not change. In future studies, the possibility of remaining $\mathrm{C}=\mathrm{C}$ could be determined with water sorption and solubility tests, and cross-link density could be measured [37].

The application of an intermediate agent, like an adhesive system, in order to penetrate into the surface irregularities and to bond with unpolymerized resin monomers of the surface, is considered essential to achieve high repair strength $[9,15,18,21]$. However, the bond strength values found in this study when the CoJet system was applied were similar to the values obtained with the use of adhesive system. According to several authors, adhesion promoters, such as enamel/dentin bonding agents and silane coupling
Fig. 3 SEM images of non-aged $\mathbf{a}$ TE and $\mathbf{b}$ FS $(\times 5,000)$, and thermocycled c TE and d FS $(\times 15,000)$.

Note the degradation of the surface in $\mathbf{c}$
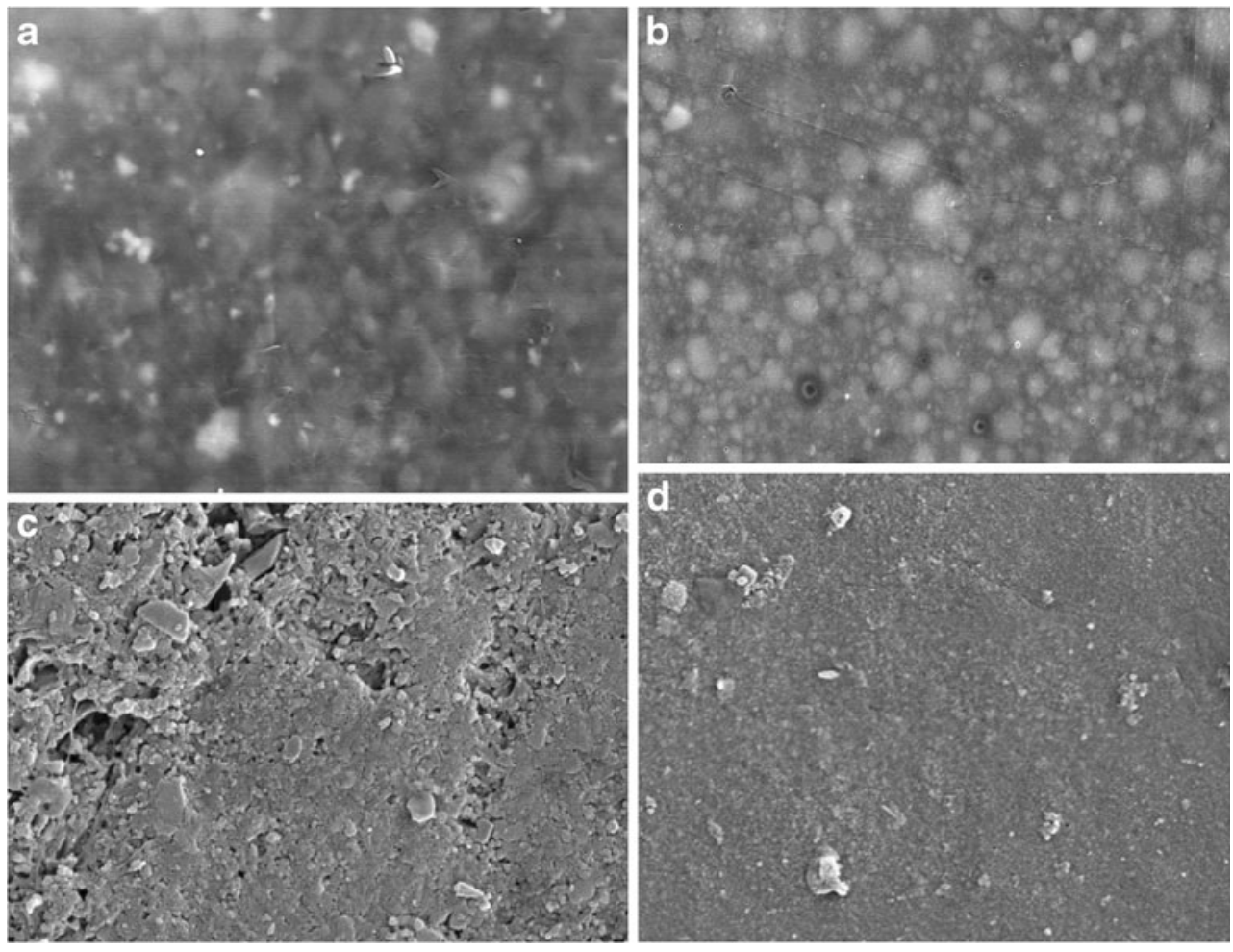
agents, may be sufficient to improve the adhesion between two RBCs $[16,22]$. Based on the results of this study, this could be partially confirmed. At least the cohesive failures in the substrate were not necessarily higher in the CoJettreated groups. The high values found with the application of adhesive systems are due to chemical bonding between the nanoparticles coated by silane and the adhesive system itself. The amount of nanosized particles on the surface of the RBC may have favoured the bonding to the adhesive system. Similarly the exchange of the adherend resin did not have a significant effect on the overall results. On the contrary, Fawzy et al. [13] showed in their study that regardless of the use of a low-viscosity resin to promote adhesion, the bond strength did not significantly improve. Also, Boushchlicher et al. reported better results with the CoJet system, with or without silane [38]. Deposition duration of air abrasion may have consequences on the results that need further investigation. In another study where MTBS was used, the CoJet system was not found to be superior to air abrasion with ordinary alumina particle [26]. However, in that study, the substrate RBCs were aged for only 9 days in water.

At this moment, bond strength studies are not decisive on suggesting the best repair protocol for RBCs. Since the substrate type seems to play a role on the stability of the bond, the clinicians are advised to keep a good track of records on the material they used. This information may be helpful when a failure is experience and a repair action needs to be taken. Clinical relevancy of bond strength data is limited, but the incidence of cohesive failures may help to predict the stability of the bond. Similarly, pre-test failures during cutting procedures may indicate weak adhesion at some areas. In this study, pre-test failures were not consistently higher in one group. For this reason, they were not considered as $0 \mathrm{MPa}$ and not involved in the bond calculations. But the frequency of pretest failures was less with the CoJet system versus adhesive systems. Clinical studies should report more on the nature of the debonding after $\mathrm{RBC}$ repairs so that the findings of in vitro studies in this field could be verified.

\section{Conclusions and clinical relevance}

Considering both the incidence of cohesive failures and the mean bond strength, repair strength seems to be more stable for the nanofilled (FS) resin composite than for the nanohybrid composite (TE) in both non-aged and aged conditions. For repair actions, surface conditioning could be accomplished with using either the corresponding adhesive resin of the resin composite only or with CoJet system, depending on the availability of the system, providing that the latter resulted in less pre-test failures. When the underlying substrate composite is unknown, the use of a dissimilar composite resin than the substrate material did not impair the repair strength.

Acknowledgments The authors acknowledge Cavex GmbH, Haarlem, the Netherlands, for the generous provision of Quadrant Anterior Shine resin composite and its adhesive resin used in this study.

Conflict of interest The authors declare that they have no conflict of interest.

\section{References}

1. Moszner N, Salz U (2001) New developments of polymeric dental composites. Prog Polym Sci 26:535-576

2. Moszner N, Klapdohr S (2004) Nanotechnology for dental composites. Int J Nanotechnol 1:130-156

3. Manhart J, Chen H, Hamm G, Hickel R (2004) Review of the clinical survival of direct and indirect restorations in posterior teeth of the permanent dentition. Oper Dent 29:481-508

4. Da Rosa Rodolpho PA, Donassollo TA, Cenci MS, Loguércio AD, Moraes RR, Bronkhorst EM, Opdam NJ, Demarco FF (2011) 22Year clinical evaluation of the performance of two posterior composites with different filler characteristics. Dent Mater 27:955-963

5. Demarco FF, Corrêa MB, Cenci MS, Moraes RR, Opdam NJM (2012) Longevity of posterior composite restorations: not only a matter of materials. Dent Mater 28:87-101

6. Blum IR, Schriever A, Heidemann D, Mjor IA, Wilson NH (2003) The repair of direct composite restorations: an international survey of the teaching of operative techniques and materials. Eur J Dent Educ 7:41-48

7. Gordan VV, Mjor IA, Blum IR, Wilson N (2003) Teaching students the repair-based composite restorations: a survey of North American dental schools. J Am Dent Assoc 134:317-323

8. Soderholm KJ, Roberts MJ (1990) Influence of water exposure on the tensile strength of composites. J Dent Res 69:1812-1816

9. Frankenberger R, Kramer N, Ebert J, Lohbauer U, Kappel S, ten Weges S, Petschelt A (2003) Fatigue behavior of the resin-resin bond of partially replaced resin based composite restorations. Am J Dent 16:7-22

10. Vankerckhoven H, Lambrechts P, van Beylen M, Davidson CL, Vanherle G (1982) Unreacted methacrylate groups on the surfaces of composite resins. J Dent Res 61:791-795

11. Swift EJ Jr, LeValley BD, Boyer DB (1992) Evaluation of new methods for composite repair. Dent Mater 8:362-365

12. Sau CW, Oh GS, Koh H, Chee CS, Lim CC (1999) Shear bond strength of repaired composite resins using a hybrid composite resin. Oper Dent 24:156-161

13. Fawzy AS, El-Askary FS, Amer MA (2008) Effect of surface treatments on the tensile bond strength of repaired water-aged anterior restorative micro-fine hybrid resin composite. J Dent 36:969-976

14. Özcan M, Barbosa SH, Melo RM, Galhano GA, Bottino MA (2007) Effect of surface conditioning methods on the microtensile bond strength of composite to composite after aging conditions. Dent Mater 23:1276-1282

15. Chan KC, Boyer DB (1983) Repair of conventional and microfilled composite resins. J Prosthet Dent 50:345-350

16. Puckett AD, Holder R, O'Hara JW (1991) Strength of posterior composite repairs using different composite/bonding agent combinations. Oper Dent 16:136-140

17. Hisamatsu N, Atsuta M, Matsumura H (2002) Effect of silane primers and unfilled resin bonding agents on repair bond strength 
of a prosthodontic microfilled composite. J Oral Rehabil 29:644648

18. Oztas N, Alacam A, Bardakcy Y (2003) The effect of air abrasion with two new bonding agents on composite repair. Oper Dent 28:149-154

19. Shen C, Mondragon E, Gordan W, Mjör IA (2004) The effect of mechanical undercuts on the strength of composite repair. J Am Dent Assoc 135:1406-1412

20. Perriard J, Lorente MC, Scherrer S, Belser UC, Wiscott HWA (2009) The effect of water storage, elapsed time and contaminants on the bond strength and interfacial polymerization of a nanohybrid composite. J Adhes Dent 11:469-478

21. Costa TRF, Ferreira SQ, Klein-Junior CA, Loguercio AD, Reis A (2010) Durability of surface treatments and intermediate agents used for repair of a polished composite. Oper Dent 35:231-237

22. Swift EJ Jr, Cloe BC, Boyer DB (1994) Effect of a silane coupling agent on composite repair strengths. Am J Dent 7:200-202

23. Özcan M (2002) The use of chairside silica coating for different dental applications: a clinical report. J Prosthet Dent 87:469-472

24. Özcan M (2003) Evaluation of alternative intra-oral repair techniques for fractured ceramic-fused-to-metal restorations. J Oral Rehabil 30:194-203

25. Sun R, Suansuwan N, Kilpatrick N, Swain M (2000) Characterisation of tribochemically assisted bonding of composite resin to porcelain and metal. J Dent 28:441-445

26. Rodrigues SA Jr, Ferracane JL, Della Bona A (2009) Influence of surface treatments on the bond strength of repaired resin composite restorative materials. Dent Mater 25:442-451

27. Rinastiti M, Özcan M, Siswomihardjo W, Busscher HJ (2011) Effects of surface conditioning on repair bond strengths of nonaged and aged microhybrid, nanohybrid, and nanofilled composite resins. Clin Oral Investig 15:625-633
28. Brendeke J, Özcan M (2007) Effect of physicochemical aging conditions on the composite-composite repair bond strength. J Adhes Dent 9:399-406

29. Özcan M, Cura C, Brendeke J (2010) Effect of aging conditions on the repair bond strength of a microhybrid and a nanohybrid resin composite. J Adhes Dent 12:451-459

30. Özcan M, Alander P, Vallittu PK, Huysmans MC, Kalk W (2005) Effect of three surface conditioning methods to improve bond strength of particulate filler resin composites. J Mater Sci Mater Med 16:21-27

31. Sideridou I, Tserki V, Papanastasiou G (2003) Study of water sorption, solubility and modulus of elasticity of light-cured dimethacrylate-based dental resins. Biomaterials 24:655-665

32. Soderholm KJ, Zigan M, Ragan M, Fischlschweiger W, Bergman M (1984) Hydrolytic degradation of dental composites. J Dent Res 63:1248-1254

33. Bagheri R, Tyas MJ, Burrow MF (2007) Subsurface degradation of resin-based composites. Dent Mater 23:944-951

34. Rinastiti M, Özcan M, Siswomihardjo W, Busscher HJ, van der Mei HC (2010) Effect of biofilm on the repair bond strengths of composites. J Dent Res 89:1476-1481

35. DeHoff PH, Anusavice KJ, Wang (1995) Three-dimensional finite element analysis of the shear bond test. Dent Mater 11:126-131

36. Souza RO, Özcan M, Michida SM, de Melo RM, Pavanelli CA, Bottino MA, Soares LE, Martin AA (2010) Conversion degree of indirect resin composites and effect of thermocycling on their physical properties. J Prosthodont 19:218-225

37. Schneider LF, Cavalcante LM, Consani S, Ferracane JL (2009) Effect of co-initiator ratio on the polymer properties of experimental resin composites formulated with camphorquinone and phenylpropanedione. Dent Mater 25:369-375

38. Bouschlicher M, Reinhardt JW, Vargas MA (1997) Surface treatment techniques for resin composite repair. Am J Dent 10:279-283 